\title{
Hypertrophy of the inferior olivary nucleus impacts perception of gravity
}

\author{
Alexander A. Tarnutzer ${ }^{1}{ }^{*}$, Antonella Palla ${ }^{1}$, Sarah Marti ${ }^{1}$, Bernhard Schuknecht ${ }^{2}$ and Dominik Straumann ${ }^{1}$ \\ ${ }^{1}$ Department of Neurology, University Hospital Zurich, Zurich, Switzerland \\ ${ }^{2}$ Medizinisch Radiologisches Institut, Zurich, Switzerland
}

\section{Edited by:}

Ji Soo Kim, Seoul National University,

South Korea

\section{Reviewed by:}

Matthew J. Thurtell, University of lowa, USA

Mark F. Walker, Case Western

Reserve University, USA

Kwang-Dong Choi, Pusan National

University Hospital, South Korea

${ }^{*}$ Correspondence:

Alexander A. Tarnutzer, Department

of Neurology, University Hospital

Zurich, Frauenklinikstrasse 26,

$\mathrm{CH}-8091$ Zurich, Switzerland.

e-mail: alexander.tarnutzer@

access.uzh.ch
Interruption of the dentato-olivary projections, interconnecting the dentate nucleus (DN) and the contralateral inferior olivary nucleus $(I O N)$, is predicted to interfere with the DN' role in estimating direction of gravity. In a patient with pendular nystagmus due to hypertrophy of the ION secondary to predominantly right-sided ponto-mesencephalic hemorrhage, perceived vertical shifted from clockwise to counter-clockwise deviations within 4 months. We hypothesize that synchronized oscillations of ION neurons induce a loss of inhibitory control, leading to hyperactivity of the contralateral DN and, as a result, to perceived vertical roll-tilt to the side of the over-active DN.

Keywords: vestibular, Guillain-Mollaret triangle, subjective visual vertical, brainstem

\section{INTRODUCTION}

Hypertrophy of the inferior olivary nucleus (ION) is an interesting phenomenon as it reflects a secondary response to an interruption of afferent connections resulting in increased and abnormally synchronized neuronal activity (Best and Regehr, 2009; Shaikh et al., 2010). Herein we report on a patient with hypertrophy of the ION secondary to ponto-mesencephalic hemorrhage and a change in the perception of earth-vertical over time. We address the role of the ION and of the dentate nucleus (DN) in the processing of vestibular input and specifically ask whether over-excitation of the DN secondary to disinhibition of the contralateral ION shifts perceived vertical into the opposite direction than previously observed for DN inhibition (e.g., due to cerebellar stroke; Baier et al., 2008).

The DN is part of an extensive network among brainstem, cerebellar, thalamic, and cortical areas involved in the integration of "graviceptive" input (Brandt, 2000; Angelaki et al., 2009) being interconnected by the central vestibular pathways (CVP). The CVP are important for posture since head position relative to gravity is signaled by one component of the vestibular system, the otolith organs. While upright, the internal representation of earth-vertical is accurate in healthy human subjects (Howard, 1982). Following unilateral or asymmetric bilateral lesions along the CVP, however, this estimate becomes biased as reflected by a tilt in the subjective visual vertical (SVV; Brandt and Dieterich, 1993). In addition to SVV tilts, vestibular tone-imbalances may lead to partial or complete ocular tilt reaction (OTR; consisting of ocular torsion, head roll, skew deviation) depending on the exact topography of the lesion (Brandt, 2000).

Anatomical studies established the pathways connecting the vestibular labyrinth to various cerebral structures. Vestibular input projects from the vestibular nuclei via bilateral ascending pathways through the medial longitudinal fascicle (MLF) to the interstitial nucleus of Cajal (INC) and through the inferior cerebellar peduncle to the cerebellar cortex. The nodulus and the ventral uvula receive vestibulo-cerebellar mossy fibers and forward these signals to the deep cerebellar nuclei. Cerebellar efferents are relayed back to the vestibular nuclei and the ION (Buttner-Ennever, 1999) and fed into ascending pathways reaching the posterolateral thalamus and ending in the temporo-peri-Sylvian vestibular cortex, where vestibular input converges with other sensory signals (Kahane et al., 2003; Dieterich and Brandt, 2008).

Previous studies identified various cerebellar structures that contribute to internal estimates of direction of gravity. These include the nodulus (Mossman and Halmagyi, 1997), the biventer lobe, and the DN (Baier et al., 2008). Clinical signs of vestibular imbalance have been linked to certain cerebellar nuclei. Specifically, lesions of the DN are associated with contraversive OTR and contralateral SVV deviations (Baier et al., 2008). The DN, however, not only receives graviceptive input from the vestibular nuclei but is also interconnected with the ION by the rubro-dentato-olivary tract ("Guillain-Mollaret triangle," Figure 1; Pearce, 2008). However, more recent work suggest that the pathway from the deep cerebellar nuclei through the superior cerebellar peduncle may not synapse to red nucleus neurons (Lapresle, 1979), but rather bypasses the red nucleus and directly projects via the central tegmental tract to the inferior olive, resulting in a dentato-olivary pathway (Matsuo and Ajax, 1979; Deuschl et al., 1990; Shaikh et al., 2010).

Lesions along the central tegmental tract may result in oculopalatal tremor (Matsuo and Ajax, 1979; Deuschl et al., 1990) and hypertrophy of the ipsilateral ION (Ruigrok et al., 1990) with its enlarged neurons developing abnormal soma-somatic gap 


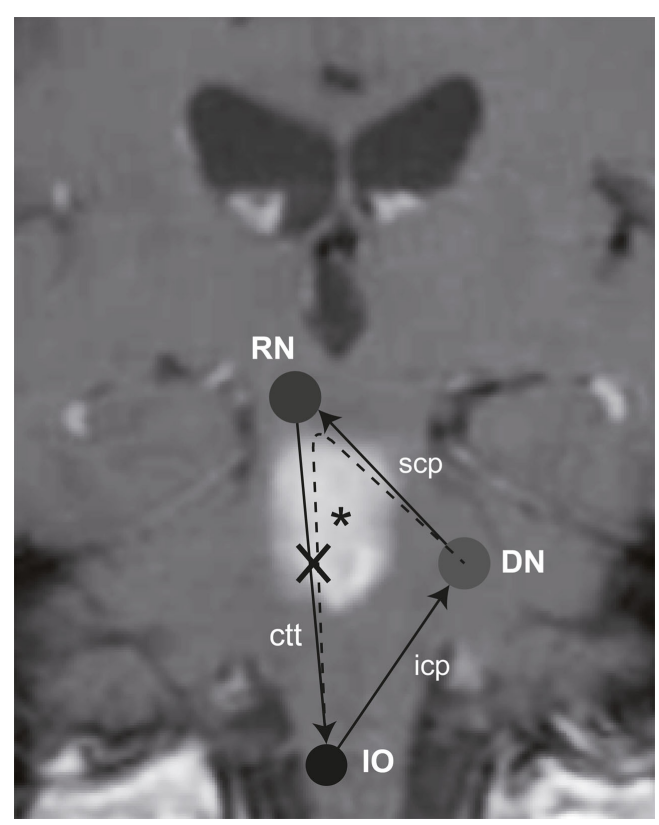

FIGURE 1 | Schematic illustration of the "Guillain-Mollaret triangle" projected on a coronal MR-image of the patient, showing the T1-hyperintense subacute ponto-mesencephalic hemorrhage (*). It is also taken into consideration, that the red nucleus may rather be bypassed (Lapresle, 1979), resulting in a direct dentato-olivary pathway (dashed line). As a consequence the right central tegmental tract (ctt) is damaged, interrupting the connection between the red nucleus (RN) and the ION. Abbreviations: icp, inferior cerebellar peduncle; scp, superior cerebellar peduncle.

junctions (Koeppen et al., 1980; De Zeeuw et al., 1998; Goyal et al., 2000). ION hypertrophy is not just a histopathological finding but can also be visualized in vivo by MR-imaging (Kitajima et al., 1994; Goyal et al., 2000). Previous work suggests that the ION is involved in coordination and timing of movements and motor learning (De Zeeuw et al., 1998). Here we evaluate whether secondary hypertrophy of the ION leads to contralateral SVV tilt indicating hyperactivity of the contralateral DN in line with the current understanding of the Guillain-Mollaret triangle.

\section{MATERIALS AND METHODS}

Informed written consent was obtained after a full explanation of the experimental procedure to the patient. The protocol was approved by a local ethics committee and was in accordance with the ethical standards laid down in the 1964 Declaration of Helsinki for research involving human subjects. SVV measurements were obtained on a motor-driven turntable (Acutronic, Jona, Switzerland) at day 16, month 4, and month 38 after symptom onset. To evaluate the SVV, the patient was asked to align a luminous arrow (time limit per trial: $15 \mathrm{~s} ; 24$ trials per position) along perceived vertical in different whole-body-roll orientations (upright, $\pm 45^{\circ}$, $\pm 90^{\circ}$ ear-down) in a random order. The arrow projection started $10 \mathrm{~s}$ after the turntable came to a full stop. The arrow starting position deviated randomly between 28 and $72^{\circ}$ clockwise (CW) or counter-clockwise (CCW) relative to true earth-vertical. For comparison, SVV adjustments (taken at angles between upright and $\pm 90^{\circ}$ every $15^{\circ}$ ) from a group of healthy normal subjects $(n=7)$ using a similar paradigm were used (Tarnutzer et al., 2009).

\section{RESULTS}

A 65-year-old male patient complaining of acute-onset gait unsteadiness presented with horizontal gaze palsy, impaired upgaze, left trigeminal hypesthesia, and left-sided sensorimotor impairment. Clinically, no signs of a (partial) OTR could be seen. An emergency head CT-scan revealed bilateral pontomesencephalic bleeding. Brain MRI obtained 14 days after symptom onset showed a predominantly right-sided pontomesencephalic dorsal hemorrhage, including the medial vestibular nuclei bilaterally, the right superior and lateral vestibular nucleus, the right (and probably also the left) MLF, and the central tegmental tract bilaterally (Figures 2A,B). No cerebellar or thalamic structures were damaged.

Subjective visual vertical measurements when upright obtained 16 days after symptom onset deviated ipsilaterally (relative to the right ION that later showed hypertrophy) by $3.0 \pm 3.0^{\circ}$ (mean \pm 1 $\mathrm{SD})$ for clockwise $(\mathrm{CW})$ arrow rotations and by $4.3 \pm 3.9^{\circ}$ for counter-clockwise (CCW) arrow rotations, whereas in all rolltilted positions roll under-compensation (SVV deviations toward the direction of body-roll) - being increased compared to deviations in healthy controls - was noted (Figure 3). A diagnostic work-up revealed no cause for the hemorrhage and the patient was discharged home.

At follow-up (month 4) the patient complained of progressive difficulty walking and fixing with the eyes. Neurologic examination revealed increased gait ataxia and new vertical-torsional pendular nystagmus. MRI showed increased signal and hypertrophy of the right ION (Figures 2C-E). SVV measurements revealed large contralateral (relative to the right ION) deviations when upright being clearly distinct for CW $\left(-20.3 \pm 10.5^{\circ}\right)$ and CCW $\left(-10.2 \pm 4.5^{\circ}\right)$ arrow rotations. For left-ear-down positions, adjustment errors remained in the same range as in the acute stage, while errors were shifted to roll over-compensation (SVV deviations away from the direction of body-roll) for right-ear-down roll.

The patient returned for regular follow-up visits and 38 months after symptom onset the SVV was re-measured. On clinical examination vertical-torsional pendular nystagmus and gait ataxia were unchanged. SVV in upright position revealed large a large trial-totrial variability of adjustment errors for both CW $\left(-3.8 \pm 24.6^{\circ}\right)$ and CCW $\left(14.1 \pm 14.0^{\circ}\right)$ arrow rotations. Again SVV errors depended on the direction of arrow rotation (Figure 3 ), but now arrow roll adjustments showed under-compensation for both directions. While roll under-compensation for left-ear-down positions were unchanged compared to previous recordings, adjustment errors for right-ear-down positions shifted back from roll over-compensation to roll under-compensation.

\section{DISCUSSION}

In this case report we describe for the first time a significant shift in the internal estimate of direction of gravity associated with hypertrophy of the ION. A roll-tilt bias in estimated direction of gravity is generally explained by an asymmetric lesion along the ascending 


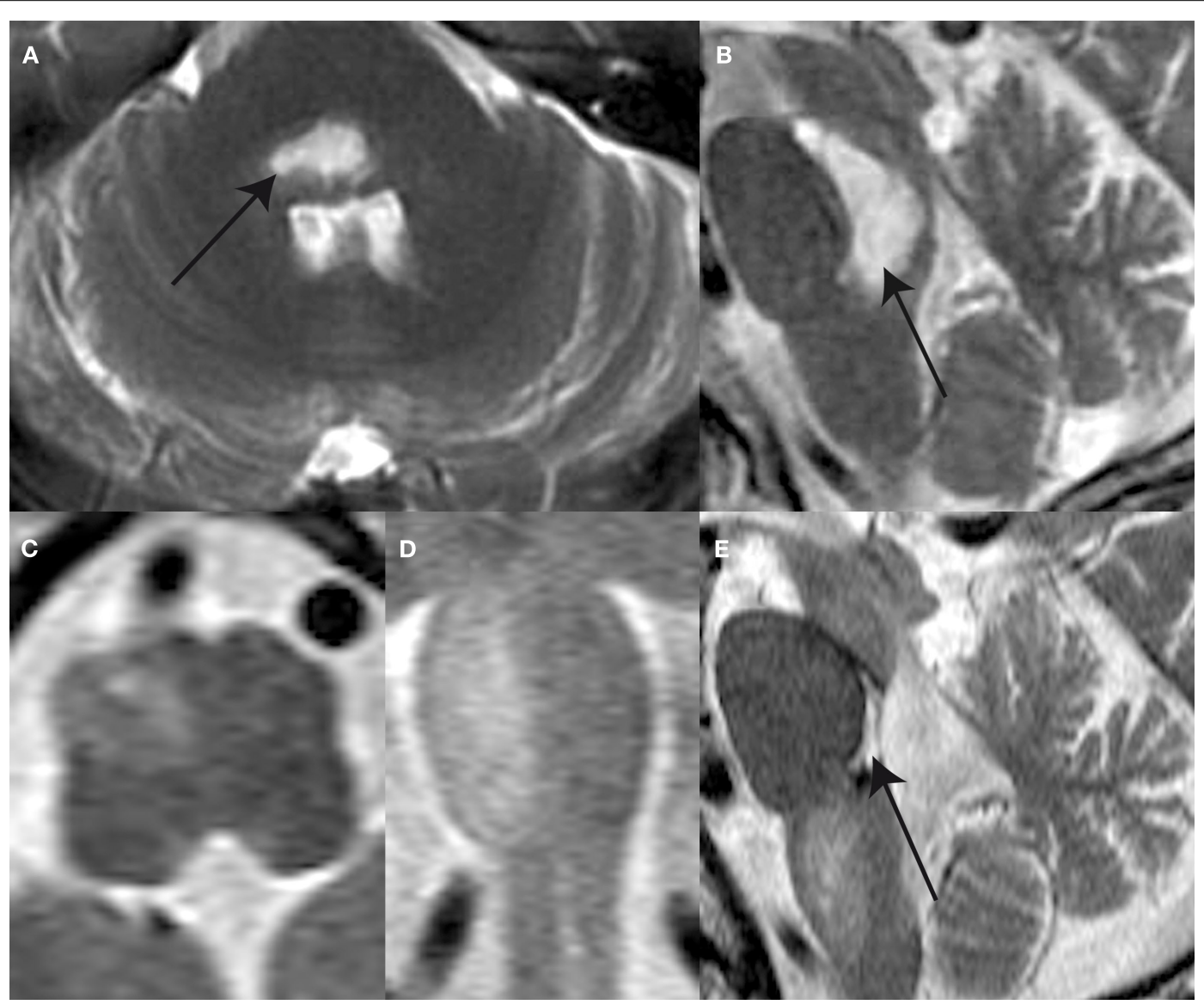

FIGURE 2 |T2-weighted MR-images shows a ponto-mesencephalic high signal hemorrhage (arrow) within the pontine and mesencephalic tegmentum anterior to the forth ventricle the axia (A) and sagittal plane (B) 14 days after symptom onset. Follow-up MR after 4 months reveals hypertrophy and prominence of the right ION as illustrated in the axial (C), coronal (D), and sagittal (E) plane images. Note the defect within pontine tegmentum (arrow) following resorption of hemorrhage. bilateral CVP (Brandt, 2000). In the acute stage, the SVV in this patient deviated rightward, i.e., toward the side of the predominant hemorrhage. Thus we assume that the loss of the right vestibular nuclei (known to result in ipsilesional SVV deviations; Dieterich and Brandt, 1993; Brandt, 2000) is the main contributor to the SVV adjustment errors measured at day 16. However, interruption of the left MLF may also contribute to the SVV tilt because MLF-lesions lead to contralateral SVV deviations (Dieterich and Brandt, 1993; Brandt, 2000). The shift in SVV from an ipsilateral (i.e., rightward) to a contralateral (i.e., leftward) SVV deviation observed at month 4 is most likely associated with hypertrophy of the right ION at that stage since the $\mathrm{DN}$ is under control of the contralateral ION (Pearce, 2008).

In the case presented here the predominantly right-sided ponto-mesencephalic hemorrhage interrupted the right central tegmental tract, which most likely explains the hypertrophy of the right-sided ION at follow-up. In the following we provide a hypothetical explanation for the switch from acute ipsilateral (with regards to the side of ION hypertrophy at follow-up) to chronic contralateral SVV deviations as a result of the hypertrophy of the ION. We conjecture that the switch in SVV tilt is a consequence of removing GABA-mediated inhibitory modulation of the ION neurons provided through the central tegmental tract (Best and Regehr, 2009). As a consequence synchronized oscillations of large groups of neurons in the ION are then sent to the cerebellar cortex and the deep cerebellar nuclei through climbing fibers (Shaikh et al., 2010) and result in a loss of the inhibitory control provided through this pathway (Leigh and Zee, 2006). This mechanism may lead to an over-excitation of the DN contralateral to the hypertrophic ION and consequently to a tilt toward the side of the over-active DN. Over-excitation of the DN leading to a shift in SVV toward the side of the stimulated DN is analogous to the reported contralesional SVV shifts in case of an inhibition (e.g., by stroke or bleeding) of the DN (Baier et al., 2008).

The relative improvement of SVV errors at month 38 despite the fact that the clinical findings (gait ataxia, vertical-torsional pendular nystagmus) persisted and MR-imaging continued to show an increased signal on T2-weighted sequences and hypertrophy of the right ION may indicate distinct mechanisms of adaptation of the involved networks. While the pendular nystagmus is governed by brainstem circuits, estimating direction of gravity is a cognitive task based on multisensory cortical integration, possibly situated 

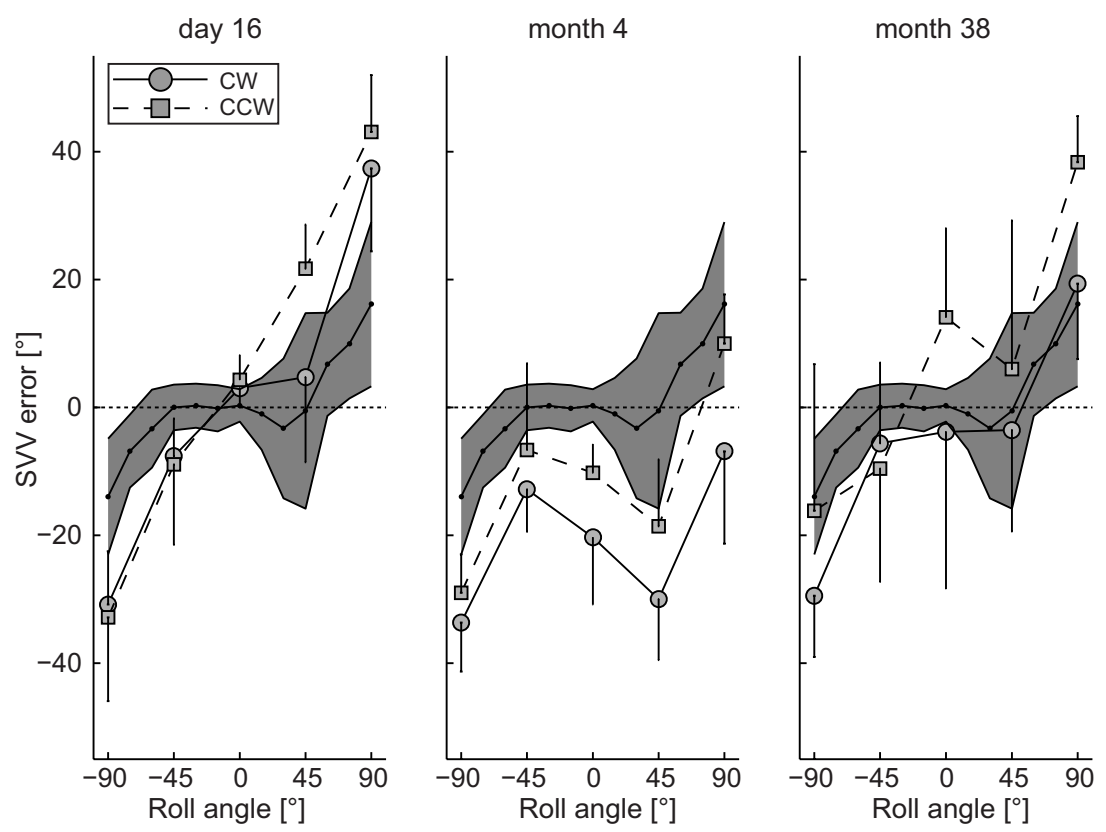

FIGURE 3 | Mean ( \pm 1 SD) SVV adjustment errors (trials with CW and CCW arrow rotations analyzed separately) plotted against whole-body-roll in the patient at day 16 , month 4 , and month 38 . For

comparison the grand mean (black dots) \pm 2 SD of SVV adjustments from seven healthy normal control subjects (data taken from Tarnutzer et al., 2009). This range covers about $95 \%$ of all values and is illustrated by the gray area.

within the temporo-peri-Sylvian vestibular cortex (Kahane et al., 2003). Based on the improvement of SVV accuracy and the ongoing nystagmus we hypothesize that long-term adaptation has led to a re-weighting of the different sensory input signals used for generating the internal estimate of direction of gravity. Thereby the otolith signal might be weighted less and consecutively the internal estimate of direction of gravity is less biased. However, SVV measurements at month 38 have to be interpreted with caution, as they varied considerably more from trial-to-trial compared to day 16 and month 4.

In summary, this case report further supports the crucial role of the DN in processing vestibular information and how it is modulated by the ION. It suggests that over-excitation of the DN by disinhibition of the ION can result in functional impairment of estimating the direction of gravity. In short, inhibition of the DN (e.g., by an ischemic cerebellar lesion including the DN) leads to

\section{REFERENCES}

Angelaki, D. E., Gu, Y., and Deangelis, G. C. (2009). Multisensory integration: psychophysics, neurophysiology, and computation. Curr. Opin. Neurobiol. 19, 452-458.

Baier, B., Bense, S., and Dieterich, M. (2008). Are signs of ocular tilt reaction in patients with cerebellar lesions mediated by the dentate nucleus? Brain 131, 1445-1454.

Best, A. R., and Regehr, W. G. (2009). Inhibitory regulation of electrically coupled neurons in the inferior olive is mediated by asynchronous release of GABA. Neuron 62, 555-565.

Brandt, T. (2000). Vertigo: Its Multisensory Syndromes. Berlin: Springer.

Brandt, T., and Dieterich, M. (1993). Skew deviation with ocular torsion: a vestibular brainstem sign of topographic diagnostic value. Ann. Neurol. 33, 528-534.

Buttner-Ennever, J. A. (1999). A review of otolith pathways to brainstem and cerebellum. Ann. N. Y. Acad. Sci. 871, 51-64.

SVV tilt away from the affected DN (Baier et al., 2008) and overexcitation of the DN - as reported here - leads to SVV tilt toward the affected DN.

\section{ACKNOWLEDGMENTS}

We thank Albert Züger, Marco Penner for technical support, and Chris Bockisch for critically reading the manuscript. Funding: this study has been supported by the Swiss National Science Foundation (grant number: 32003B_130163/1 and 3200B0-105434), Berne, Switzerland; the Betty and David Koetser Foundation for Brain Research, Zurich, Switzerland; and the Center of Integrative Human Physiology, University of Zurich, Switzerland. None of these sponsors was involved in the study design; in the collection, analysis, and interpretation of the data; in the writing of the report; and in the decision to submit the paper for publication.

De Zeeuw, C. I., Simpson, J. I., Hoogenraad, C. C., Galjart, N., Koekkoek, S. K., and Ruigrok, T. J. (1998). Microcircuitry and function of the inferior olive. Trends Neurosci. 21, 391-400.

Deuschl, G., Mischke, G., Schenck, E., Schulte-Monting, J., and Lucking, C. H. (1990). Symptomatic and essential rhythmic palatal myoclonus. Brain 113(Pt 6), 1645-1672.

Dieterich, M., and Brandt, T. (1993). Ocular torsion and tilt of subjective visual vertical are sensitive brainstem signs. Ann. Neurol.33, 292-299.

Dieterich, M., and Brandt, T. (2008). Functional brain imaging of peripheral and central vestibular disorders. Brain 131, 2538-2552.

Goyal, M., Versnick, E., Tuite, P., Cyr, J. S., Kucharczyk, W., Montanera, W., Willinsky, R., and Mikulis, D. (2000). Hypertrophic olivary degeneration: metaanalysis of the temporal evolution of MR findings. AJNR Am. J. Neuroradiol. 21, 1073-1077. 
Howard, I. P. (1982). Human Visual Orientation. New York: Wiley.

Kahane, P., Hoffmann, D., Minotti, L., and Berthoz, A. (2003). Reappraisal of the human vestibular cortex by cortical electrical stimulation study. Ann. Neurol. 54, 615-624.

Kitajima, M., Korogi, Y., Shimomura, O., Sakamoto, Y., Hirai, T., Miyayama, H., and Takahashi, M. (1994). Hypertrophic olivary degeneration: MR imaging and pathologic findings. Radiology 192, 539-543.

Koeppen, A. H., Barron, K. D., and Dentinger, M. P. (1980). Olivary hypertrophy: histochemical demonstration of hydrolytic enzymes. Neurology 30, 471-480.

Lapresle, J. (1979). Rhythmic palatal myoclonus and the dentato-olivary pathway. J. Neurol. 220, 223-230.
Leigh, R. J., and Zee, D. S. (2006). The Neurology of Eye Movements, 4th Edn. New York: Oxford University Press.

Matsuo, F., and Ajax, E. T. (1979). Palatal myoclonus and denervation supersensitivity in the central nervous system. Ann. Neurol. 5, 72-78.

Mossman, S., and Halmagyi, G. M. (1997). Partial ocular tilt reaction due to unilateral cerebellar lesion. Neurology 49, 491-493.

Pearce, J. M. (2008). Palatal myoclonus (syn. palatal tremor). Eur. Neurol.60, 312-315.

Ruigrok, T. J., De Zeeuw, C. I., and Voogd, J. (1990). Hypertrophy of inferior olivary neurons: a degenerative, regenerative or plasticity phenomenon. Eur. J. Morphol. 28, 224-239.
Shaikh, A. G., Hong, S., Liao, K., Tian, J., Solomon, D., Zee, D. S., Leigh, R. J., and Optican, L. M. (2010). Oculopalatal tremor explained by a model of inferior olivary hypertrophy and cerebellar plasticity. Brain 133, 923-940.

Tarnutzer, A. A., Bockisch, C., Straumann, D., and Olasagasti, I. (2009). Gravity dependence of subjective visual vertical variability. J. Neurophysiol. 102, 1657-1671.

Conflict of Interest Statement: The authors declare that the research was conducted in the absence of any commercial or financial relationships that could be construed as a potential conflict of interest.
Received: 05 March 2012; accepted: 24 April 2012; published online: 11 May 2012.

Citation: Tarnutzer AA, Palla A, Marti $S$, Schuknecht $B$ and Straumann $D$ (2012) Hypertrophy of the inferior olivary nucleus impacts perception of gravity. Front. Neur. 3:79. doi: 10.3389/fneur.2012.00079

This article was submitted to Frontiers in Neuro-otology, a specialty of Frontiers in Neurology.

Copyright (C) 2012 Tarnutzer, Palla, Marti, Schuknecht and Straumann. This is an open-access article distributed under the terms of the Creative Commons Attribution Non Commercial License, which permits non-commercial use, distribution, and reproduction in other forums, provided the original authors and source are credited. 